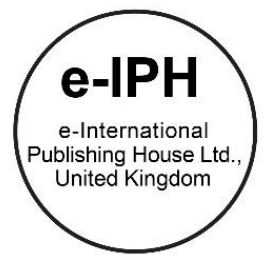

\title{
Health Screening Behaviour among Female Urban Dwellers
}

\author{
Nik Nairan Abdullah*, Mohd Shahril Ahmad Saman, Nor Aini Mohd Noor \\ Population Health \& Preventive Medicine, Faculty of Medicine, Universiti Teknologi MARA(UiTM), Sungai Buloh and 47000, Malaysia
}

\begin{abstract}
An ageing population is a public health challenge, affects most countries. Health screenings are able to detect diseases at the earliest stage. $\mathrm{A}$ cross-sectional study in December 2014 conducted among 643 older women who randomly interviewed using structured questionnaire from two urban governmental health centres in Malaysia. Aims of the study were to describe health screening services behaviour and health care accessibility among women aged 50 and above. Factors such as living arrangement and age played important roles in health screening execution among older female community dwellers. Advocacy on health screening is vital as to reduce the morbidity and mortality among them.

(C 2016. The Authors. Published for AMER ABRA by e-International Publishing House, Ltd., UK. Peer-review under responsibility of AMER (Association of Malaysian Environment-Behaviour Researchers), ABRA (Association of Behavioural Researchers on Asians) and cE-Bs (Centre for Environment-Behaviour Studies, Faculty of Architecture, Planning \& Surveying, Universiti Teknologi MARA, Malaysia.
\end{abstract}

Keywords: Older people; female; urban; health screening

\section{Introduction}

A better health status is associated with a longer life expectancy (Alina et al., 2013). Malaysia, being a developing country is witnessing an increasing proportion of older people. As reported by Mohammad and Abbas (2012), the chronological definition for elderly in Malaysia are those aged 60 years old and above. Older population aged 60 and over has increased from $3.1 \%$ (1970) to $4.2 \%$ (2000). In 2020, the older population in Malaysia is projected to be $7.3 \%$, thus, this will turn Malaysia to ageing population (Wan-lbrahim \& Zainab, 2014).

Population ageing is typically caused by the demographic transition. Population transition from high rates of both fertility and mortality to the lower rate of fertility and mortality, rising life expectancy and increasingly, by the ageing of baby boomer generation (Mohammad \& Abbas, 2012; Somenahalli \& Shipton, 2013; Wan-Ibrahim \& Zainab, 2014). Good health conditions can influence mortality, fertility and life expectancy while demographic transition that have different effects on economic growth (Alina et al., 2013). Health is strictly related to the demographic structure of the population and determines both human capital stock and accumulation, which are the essential element for economic development (Alina et al., 2013). Promoting a good health status of a population is an important objective of a nation as it has economic implications. Longevity influences economic growth,

\footnotetext{
* Corresponding author. Tel.: +60-03-61267214 ; fax : +60-03-61267069

E-mail address: niknairan @yahoo.com
} 
but a longer life expectancy with lower fertility and adult mortality rates will result in a higher proportion of the elderly population. These will cause an adverse impact on economic growth (Alina et al., 2013).

This demographic change has considerable implications for health care that is closely related to modernization. The urbanization that is taking place may have resulted in the dropping rates of fertility while improved health technology has reduced mortality rates. With an increasing proportion of the elderly population, the numbers of those with chronic and complex medical problems will also increase. Chronic diseases are the main causes of morbidity and mortality in developed and developing countries (Halpin et al., 2010). These chronic diseases - including heart disease, cancer, stroke, diabetes, and respiratory diseases share the same major risk factors. The known risk factor apart from genetic predisposition includes unhealthy lifestyle modifications. Tobacco use, unhealthy diet and physical inactivity are some of the lifestyle changes. (Halpin et al., 2010). It may contribute to the sharp rise in the prevalence of chronic diseases. Also to the effect of aging, the number of cancer patients will also raise indisputably.

Therefore, it is common to observe an increasing percentage of general practice consultations and acute hospital admissions for the elderly. In Malaysia, older people have an average of six consultations yearly as compared to the national average of 2.3 visits for the younger people (Forsyth \& Chia, 2009). The rise in the number of medical consultation and hospital admission among the elderly will undoubtedly impinge on the health care cost. Lack of access to preventive care according to Halpin et al. (2010) is one of the factors that may add to the escalating prevalence of chronic diseases. Preventive care consisted of health screening. Screening lies in the secondary level of prevention, and it is clearly documented that it can avert thousands of deaths of relatively young women globally. The aim of screening is to detect the early stage of a disease when there is no symptom so that prompt intervention can be applied. In Malaysia, preventive health screening is available at private and governmental health facilities. The types of screenings are divided into chronic diseases and cancer screening. A basic screening detects common chronic illnesses such as hypertension, diabetes, and hypercholesterolemia. A person who is found to be overweight or obese during screening will be referred for weight management consultation.

Examples of common cancer screening for female are Pap smear to screen for cervical cancer and mammogram for breast cancer. Early management that includes medication and surgical intervention will reduce the morbidity and mortality that resulted from cancer. A comprehensive health care ranges from primary to tertiary prevention is an utmost importance for the overall wellbeing of an individual. Since the health care cost spiralling up in the last decade, it is more cost-effective to expand the health screening services given enhancing compressed morbidity phenomenon. Although females have longer life expectancy compared to male, they are subjected to more screening modalities because women are prone to develop cancers of the reproductive organs due to the influence of various hormones. Furthermore, there is a scarcity of published data on chronic diseases among the urban older female community. There has been a great emphasis on institutional care as compared to community studies. Institutional care incurs the most financial cost and some Western countries such as Australia are going towards community- based care.

Objectives of the study were to explore the behavior of older female urban dwellers, aged 50 and above on the utilization of health screening services and health care accessibility. Also, the distribution of medical disorders and the associations between sociodemographic factors and health screening were determined.

\section{Methodology}

This study is the pilot result of a cross-sectional study in late December 2014.It was conducted among 643 women who were randomly selected from two governmental health centres. These clinics were located in the urban areas which were Kepong and Sungai Buloh. Women aged 50, and above who were not bound to wheelchair were screened for cognitive impairment using the Elderly cognitive assessment questionnaire (ECAQ). It is important to screen them before administering the questionnaire to ensure the reliability of data. ECAQ is a validated questionnaire to screen any mild cognitive impairment among the elderly. Those who were unable to attain a score of six and above were excluded from the study. A total of 643 women passed the screening test and participated in the study. A written consent was then obtained from the participants. Participants were ensured of the confidentiality of the information given. Trained research assistants interviewed the respondents using the structured questionnaire.

\subsection{Study instruments}


The questionnaire was divided into three sections that consist of Part A - demographic details, self-reported chronic medical disorders and living arrangement; Part B- health care accessibility (distance from home to health centre including the mode of transportation); Part C- The utilization of comprehensive basic screening comprised of blood pressure, blood lipid, blood glucose, anthropometric measurement, bone scan and common cancer screening. The anthropometric measurement was divided into Body Mass Index, waist circumference and waist to hip ratio.The cancer screening consisted of cervical cancer screening (Pap smear) and three types of breast screening that were breast-self, clinical breast examination and mammogram. Bone scan and mammogram screening services are not available in the health centre. Nevertheless, the medical doctor in the health facility who is the gatekeeper has the obligation to refer patients to the hospital for such screening.

Respondents who performed all the types of screening mentioned above were defined as performed a complete comprehensive basic screening. Any missed form of screening will be categorized as partial screening. Those who did not perform any of the screening are considered as 'not performing'. Statistical Package for Social Sciences (SPSS) was applied for data analysis. Descriptive analysis was conducted to describe the study population characteristics. Further analyses involved the calculation of the crude odds ratio (COR), adjusted odd ratio (AOR) and $95 \%$ confidence interval (Cl) using logistic regression.

\section{Results and Discussions}

Table 1. Study population characteristics and screening behaviour

\begin{tabular}{lllll}
\hline Socio-demographic factors & Frequency $(\mathrm{n}) \mathrm{N}=643$ & $\%$ & \multicolumn{2}{l}{ Basic screening } \\
\hline Age & & & No & Yes \\
$50-60$ & 379 & 58.94 & $30(7.9)$ & $349(92.1)$ \\
$61-70$ & 193 & 30.02 & $7(3.6)$ & $186(96.4)$ \\
71 and above & 71 & 11.04 & $2(2.8)$ & $69(97.2)$ \\
Ethnicity & & & & \\
Malay & 459 & 71.38 & $21(4.6)$ & $437(95.4)$ \\
Chinese & 101 & 15.71 & $9(8.9)$ & $92(91.1)$ \\
Indian & 71 & 11.04 & $7(9.9)$ & $64(90.1)$ \\
Others & 12 & 1.87 & $2(16.7)$ & $10(83.3)$ \\
Marital Status & & & & \\
Single & 23 & 3.58 & $2(8.7)$ & $21(91.3)$ \\
Married & 515 & 80.22 & $33(6.4)$ & $482(93.6)$ \\
Divorced/Widowed & 105 & 16.2 & $4(3.8)$ & $101(96.2)$ \\
Educational level & & & & \\
Never formal education & 75 & 11.66 & $6(8)$ & $69(92)$ \\
Primary level & 212 & 32.97 & $15(7.1)$ & $197(92.9)$ \\
Secondary level & 307 & 47.74 & $14(4.6)$ & $293(95.4)$ \\
Tertiary level & 49 & 7.62 & $4(8.2)$ & $45(91.8)$ \\
Living arrangement & & & & \\
Living alone & 29 & 4.52 & $4(13.8)$ & $25(86.2)$ \\
Living with spouse & 124 & 19.28 & $3(2.4)$ & $121(97.6)$ \\
Living with family members/others & 490 & 76.20 & $32(6.5)$ & $458(93.5)$ \\
Accompanying person to health care centre & & & & \\
None( alone) & 159 & $9(5.7)$ & $150(94.3)$ \\
\hline
\end{tabular}


Abdullah, N.N., et al. / $6^{\text {th }}$ AicE-Bs2015Barcelona, Spain, 30 Aug.- 04 Sep. 2015 / E-BPJ, Maiden Issue, 1(1) June 2016 (pp.117-122)

\begin{tabular}{lllll}
\hline $\begin{array}{l}\text { Family members } \\
\text { Others }\end{array}$ & 469 & 72.94 & $27(5.8)$ & $442(94.2)$ \\
Number of medical disorders & 15 & 2.33 & $3(20)$ & $12(80)$ \\
None & & & & \\
$1-2$ & 95 & 14.62 & $10(10.5)$ & $85(89.5)$ \\
3 and more & 412 & 64.23 & $22(5.3)$ & $390(94.7)$ \\
\hline
\end{tabular}

A total of 643 women who attended two urban health centres completed the questionnaire. The mean age of the participants was $60.09+7.96$ years old, range $(50-88)$ years old (refer Table 1$)$. The majority of them were Malays $(71.38 \%)$, followed by Chinese (15.71\%) and Indians (11.04\%).Unlike in other countries, one of the legacies from previous British colonization in the past was organised, centralised health delivery system. The planned ideal distance between health facilities is 5 kilometers for Peninsula Malaysia and between homes to health centres is 2.5 kilometers. The health centres were accessible well from their homes. From this study, the mean distance from their homes to the governmental health centre was $7.64 \pm 6.84 \mathrm{~km}$, was not far deranged from the national plan. This was coherent to study noted by Somenahalli and Shipton (2013) where they found that elderly population are less prone to travel long distances and in long period, their access to health services is often less than of younger people. The distribution of elderly is explained better by the area's access to services than socioeconomic factors (Somenahalli \& Shipton., 2013). Therefore, we do not experience much distance problem in urban areas except for the transportation. Only small percentage took public transport (16.95\%) to the health centre in which only $5.4 \%$ took the bus. A study done by Abbas and Saruwono (2012) noted that only half (58\%) elderly who stayed in own house expressed that it was easy to engage with public transport. There are few reasons that may contribute to the poor public bus usage. The irregularity of bus interval, reduced coverage and buses not being disabled friendly are some of the drawbacks. Moreover, one-quarter of them had a mobility disability; the condition caused hindrance for the public transportation utilisation. According to Prince et al. (2015), access and coverage are especially poor in older people. Hence, efforts should be made to create elderly-friendly infrastructure and environment at the health facilities.

For elderly, design features for the healthcare buildings should be unobtrusive and easy to incorporate, allow flexibility and the ability to function whatever their condition and ability (Mohammad \& Abbas., 2012). Structures such as the ramp, even step, railings at the stairs and toilet were constructed. The walkway and the stairs were well lit and marked. Fast lane priority is given for those aged more than 60 years old. This study showed that our society still hold on to our culture that is family-centric in nature where only $25 \%$ of older women went to the health centre on their own. Similarly noted by Abedi et al. (2010) where family support and health care services are the main influential factors in elderly health care. Most of them were brought in by their family members while only one-third $(34.06 \%)$ drove themselves to the health care facilities. Elderly with higher level information and awareness leads to greater interest to change their lifestyle (Abedi et al., 2010). With that interest, motivation becomes the most important component in the intrapersonal inner representation for health screening execution. There is a tendency of decreasing of hardiness levels in an undiagnosed subject with symptoms of chronic disease as compared to those who were healthy and those with diagnosed chronic diseases (Nikolaeva \& Elnikova., 2014). Although there was a remarkable high proportion of $80.13 \%$ older female urban dwellers had ever undergone basic health screening, the majority $(59.72 \%)$ performed it partially. Among the health screening modalities that they did not perform were the Pap smear $(60 \%)$, breast screening (47\%) and bone scan (81.5\%). Abdullah et al. (2013) found that embarrassment and cost were influential factors of health screening execution among the population. According to Meneze et al. (2015), based on Health Belief Model, main barrier to health seeking behavior were physical environment, availability, affordability, lack of familiarity with the health care system and lack of information available. Apart from that, long waiting time and the incompatible working hours of clinics were the hindrance factors. Also noted inattentive doctors were among known barriers.

In term of the number of medical disorders, $64.07 \%$ urban dwellers reported having one or two medical disorders. A similar pattern was seen in the rural areas. Most of the elderly had one illness while a smaller percentage had two or three diseases. About $60.1 \%$ had been diagnosed with chronic diseases with a smaller percentage had two or three illnesses (Shahar et al., 2001). Most of these women have hypertension (68.43\%) followed by diabetes mellitus (47.74\%), osteoarthritis $(21.93 \%)$ and other medical disorders. This is by local studies documented hypertension as the most common medical illness (Prince et al., 
2015; Shahar et al., 2010; Sherina et al., 2004). The high prevalence of hypertension was similar to the global data according to Halpin et al. (2010).

The second most prevalent disorder was diabetes mellitus. Neuroendocrine theory of aging identifies clusters of conditions (hypertension, diabetes type 2, obesity, dyslipidemia, menopause, depression, vascular cognitive impairment, impairment of immune system and cancer. The manifestations of metabolic syndrome are high cholesterol and triglyceride levels and central obesity with the majority reported with antecedents of cardiovascular diseases.Metabolic syndrome represents a public health problem due to severe multisystem consequences it induces, affecting a large group of people especially older population (Ciucurel \& Iconaru., 2012). In contrary to Shahar et al. (2001)'s finding, the major chronic illness reported among the elderly were joint pains $(45.0 \%)$. This discrepancy was due to joint pains were considered as symptoms not as a disorder as osteoarthritis.

It is understood that the older people with medical disorders were more likely to visit the clinic compared to those without. The mean visit made to these facilities was $2.58 \pm 2.96$ within the six months duration. If the respondent has more than one medical disorder, it is reasonable for them to make frequent visits to the health centres. However, the visits may be due to blood test investigation alone that needs to be performed at regular intervals. In regards to health screening among the older population (Table 2), women aged between 61 and 70 years old has significant higher odds of being screened, (AOR=2.84,95\% $\mathrm{Cl}$ : 1.16,6.96). One of the few reasons that may explain for this is opportunistic screening. Once a health practitioner has diagnosed a patient with chronic medical disorder, it is prudent to screen the patient for other health problems. This practice is known as opportunistic screening. It is a type of screening that is confined to the act of screening conducted by the health practitioner to a patient who consulted for some other purpose, for example taking a blood pressure when complaining of a viral fever.

Table 2. Factors associated with performing screening in the univariate and multivariate analysis

\begin{tabular}{lll}
\hline Factors & Crude Odds ratio $(95 \% \mathrm{Cl})$ & Adjusted Odds Ratio(95\% Cl) \\
\hline Age & & \\
$50-60$ & Reference & Reference \\
$61-70$ & $2.89(1.12,7.42)$ & $2.84(1.16,6.96)$ \\
71 and above & $4.78(0.96,23.78)$ & $4.45(0.99,19.98)$ \\
Living arrangement & Reference & Reference \\
Living Alone & $7.02(1.19,41.36)$ & $7.31(1.45,36.97)$ \\
Living with Spouse & $2.04(0.60,9.62)$ & $3.07(0.92,10.20)$ \\
Living with Family Members & & \\
\hline
\end{tabular}

Adjusted odds ratios for ethnicity, marital status, education, accompanying person, insurance and medical disorders as confounding variables

In the multivariate analysis, it seems that living arrangement and age have huge influences on the utilisation of the health screening. Apparently, it showed that older women who live with their spouses had more likely to perform screening compared to those living alone (AOR=7.31,95\% Cl: 1.45,36.97). This evidence supports the importance of spousal influences in the decisionmaking (Carlssons et al., 2012).

There were few limitations of the study occurred in this study. The respondents were taken from two governmental health centers, rather from the population at large. Also, the medical diagnosis was based on a self-reporting diagnosis of the doctor by the respondents.

As this study able to shed some light on the health screening behavior among female urbanites, there were a lot more issues needed to be explored in great details. As for the direction of future research, there are needs to do qualitative research to address this limitation. Also, a study on the primordial factors such as health policy and environmental factors need to be pursued in the future study. 


\section{Conclusion and Recommendation}

Even though a huge proportion of older female urban folk came to health centers for health screening, less than one-fifth of them engaged public transport. A little proportion of five percent took the public bus as the mode of transportation. These were due to the irregularity of bus interval, reduced coverage and mobility disability.

Less than half of them performed complete comprehensive health screening. Those living with a spouse were more likely to perform health screening as compared those staying alone. Below are some of the recommendations as to improve the proportion of complete comprehensive health screening:

- Prevent further mobility disability among elderly

- Public bus improvement in term of shorter interval and disable friendly.

- Tackle the embarrassment of Pap smear and breast examination issues.

- Subsidize the cost of tests such as mammogram and bone scan.

Creating an accessible environment is an essential measure to strengthen the health screening behavior of the elderly population.

\section{Acknowledgements}

We wish to acknowledge Gombak health district office for the opportunity to conduct the survey at their vicinities and also medical students of Universiti Teknologi MARA(UiTM).

\section{References}

Abbas, M. Y., \& Saruwono, M. (2012). Our 'golden'citizens with 'golden'facilities? Procedia-Social and Behavioral Sciences, 49, 127-146.

Abdullah, N. N., Al-Kubaisy, W., \& Mokhtar, M. M. (2013). Health behaviour regarding cervical cancer screening among urban women in Malaysia. ProcediaSocial and Behavioral Sciences, 85, 110-117.

Abedi, H., Mostafavidarani, F., \&Riji, H. M. (2010). The elderly perception and views on their health-facilitating and inhibiting factors in elderly health care in Iran: a qualitative study. Procedia-Social and Behavioral Sciences, 5, 2222-2226.

Alina, C., Donatella, F., Raffaella, M., \& Nicola, M. (2013).Demographics and health in the Eu27 development process. Procedia-Social and Behavioral Sciences, 93, 704-708.

Carlsson, F., He, H., Martinsson, P., Qin, P., \& Sutter, M. (2012). Household decision making in rural China: Using experiments to estimate the influences of spouses. Journal of Economic Behavior\& Organization, 84(2), 525-536.

Ciucurel, C., \&Iconaru, E. I. (2012).Association between metabolic syndrome and depression in elderly. Procedia-Social and Behavioral Sciences, $33,994-997$.

Forsyth, D. R., \& Chia, Y. C. (2009). How should Malaysia respond to its ageing society. Med J Malaysia, 64(1), 47.

Halpin, HA., Morales-Suárez-Varela, MM., Martin-Moreno, J.M.(2010).Chronic disease prevention and the new public health. Public Health Reviews,32,120-154.

Maneze, D., DiGiacomo, M., Salamonson, Y., Descallar, J., \& Davidson, P. M. (2015). Facilitators and barriers to health-seeking behaviours among filipino migrants: inductive analysis to inform health promotion. BioMed Research International.

Mohammad, N. M. N., \& Abbas, M. Y. (2012). Elderly Environment in Malaysia: Impact of Multiple Built Environment Characteristics. Procedia-Social and Behavioral Sciences, 49, 120-126.

Nikolaeva, E., \&Elnikova, O. (2014). Attitude to the diseases of the people with different health levels. Procedia-Social and Behavioral Sciences, 159, 96-100.

Prince, M. J., Wu, F., Guo, Y., Robledo, L. M. G., O'Donnell, M., Sullivan, R., \& Yusuf, S. (2015). The burden of disease in older people and implications for health policy and practice. The Lancet, 385(9967), 549-562.

Shahar, S., Earland, J., Abd Rahman, S.(2001). Social and health profiles of rural elderly Malays. Singapore Med J , 42(5),208-213.

Sherina, M. S., Rampal, L., \& Mustaqim, A. (2004). Factors associated with chronic illness among the elderly in a rural community in Malaysia. Asia-Pacific Journal of Public Health, 16(2), 109-114.

Somenahalli, S., \& Shipton, M. (2013). Examining the distribution of the elderly and accessibility to essential services. Procedia-Social and Behavioral Sciences, 104, 942-951.

Wan-Ibrahim, W. A., \& Zainab, I. (2014). Some Demographic Aspects of Population Aging in Malaysia. World Applied Sciences Journal, 30 (7), 891-894. 